\title{
Robotic Forceps with a Flexible Wrist Joint Made of Super Engineering Plastic
}

\author{
Dongbo Zhou, ${ }^{1}$ Kiyoshi Sanada, ${ }^{2}$ and Daisuke Haraguchi ${ }^{1 *}$ \\ ${ }^{1}$ Institute of Innovative Research, Tokyo Institute of Technology, \\ 4259 Nagatsuta-cho, Midori-ku, Yokohama, 226-8503, Japan \\ ${ }^{2}$ RIVERFIELD Inc.,20 Samon-cho, Shinjuku-ku, Tokyo, 160-0017, Japan
}

(Received October 13, 2019; accepted November 25, 2019)

Keywords: robotic forceps, flexible wrist, super engineering plastic, continuum joint

A pair of miniaturized robotic forceps with a flexible wrist joint has a problem of a trade-off relationship between rigid and flexible characteristics of the joint with metal parts. Regarding the problem, in this paper, we present a pair of robotic forceps in which the flexible wrist is made of super engineering plastic that is widely used for medical instruments because of its superior heat resistance, chemical stability, and mechanical strength. A prototype flexible wrist part is designed using polyetheretherketone (PEEK) with 12 machined slits, and the bending and grasping motions of the forceps are realized by wire actuation. The performance evaluation results show that the PEEK flexible joint can maintain its bending range even if an axial compressive force is exerted. It is durable to a compressive force of $30 \mathrm{~N}$, and the relationship between the compression extent and the compression force is linear. The PEEK flexible joint is durable to bending up to 10000 times without markedly changing its bending mechanical property. Finally, it is rigid enough to output a force more than $1.2 \mathrm{~N}$ from the forceps gripper to the environment. Experimental results in this study indicate that the developed forceps has a basic feasible performance for robotic surgery.

\section{Introduction}

Multiple degrees of freedom (DOFs) are an essential requirement of robotic forceps used in a robotic minimally invasive surgical system. In this paper, we focus on the mechanism of the distal bending joints (wrist part) of robotic forceps.

Flexible and continuum joints are superior to rigid link joints in mechanical simplicity and miniaturization because the number of parts in a flexible joint is smaller, and extra small parts such as shafts, bearings, and pulleys are not needed. Ding et al. developed an insertable robot effector platform with the multi-backbone continuum mechanism to realize the bending motion. ${ }^{(1)}$ Haraguchi et al. proposed a pair of pneumatically driven multi-DOF forceps using a machined spring combined with a backbone structure of a NiTi superelastic wire. ${ }^{(2)} \mathrm{Hu}$ et al. developed a flexible suturing robot using two helical-spring bending sections that respectively rotate in pitch and yaw directions. ${ }^{(3)}$

*Corresponding author: e-mail: haraguchi.d.aa@m.titeh.ac.jp https://doi.org/10.18494/SAM.2020.2655 
Many of these flexible and continuum joints employed wire-actuation mechanisms to realize the bending motion and showed great flexibility. However, large tensions of actuation wires will generate excessive compression of the flexible joint. The axial stiffness should be observed. The above-mentioned flexible and continuum joints are mainly made of metal materials such as titanium alloy and stainless steel. It is difficult for the metallic bending joints to improve their performance in terms of both compression rigidity and bending flexibility.

Apart from elastic and continuum elements, other research groups use "quasi-flexible" joints consisting of accumulating rigid disks ${ }^{(4,5)}$ or sections $^{(6)}$ to increase the axial stiffness. However, the bending direction and bending range are limited at each section, so the mechanism connecting the rigid disks or sections increases the length of the flexible joint, which in turn increases the bending radius of the joint, reducing its bending dexterity.

However, it is still necessary for the wrist joint in robotic forceps to meet the dual requirements of rigid and flexible characteristics, especially for executing a laparoscopic operation in a narrow space. Therefore, another material is expected to be used in the continuum joint instead of metals. Super engineering plastics (SEPs) are often used in mechanical parts facing the workload in both axis and bending directions (for example, flexible couplings made of polyacetal materials). Furthermore, the SEPs often have superior heat resistance, chemical stability, and so forth. These advantages make the SEPs suitable for medical instruments used in special applications such as insulation, sterilization, weight reduction, and nonmagnetism.

Therefore, the authors propose a pair of robotic forceps, for which a SEP was chosen as the material for the flexible joint because of its following three advantages. First, a SEP joint is more flexible in bending than metal joints while keeping moderate axial stiffness. Second, a SEP joint is reasonably applicable to medical energy devices such as electric knives because of its electrical insulation. Third, low-cost fabrication can be realized by injection molding in the mass production stage.

We herein present the design and prototype of the robotic forceps with a SEP flexible wrist joint and its practical performance evaluation. In Sect. 2, we describe the SEP flexible joint structure and actuation mechanism of the robotic forceps. Section 3 shows some experimental results of the mechanical characteristics and durability of the SEP flexible joint for a practical evaluation of its performance.

\section{Materials and Methods}

\subsection{Design and mechanism of the robotic forceps}

Figure 1 shows our robotic forceps. It mainly comprises the forceps and drive units. The forceps unit is connected to the drive unit through the actuator adaptor, which is designed for transmitting the motion of the actuators in the drive unit to the forceps unit, and for easily separating the sterilized and unsterilized parts. The forceps has a flexible wrist with two-DOF bending motion and a gripper. The diameters of the forceps shaft and flexible wrist are both $4.5 \mathrm{~mm}$. The drive unit, which has five pneumatic cylinders with position sensors, conducts a 


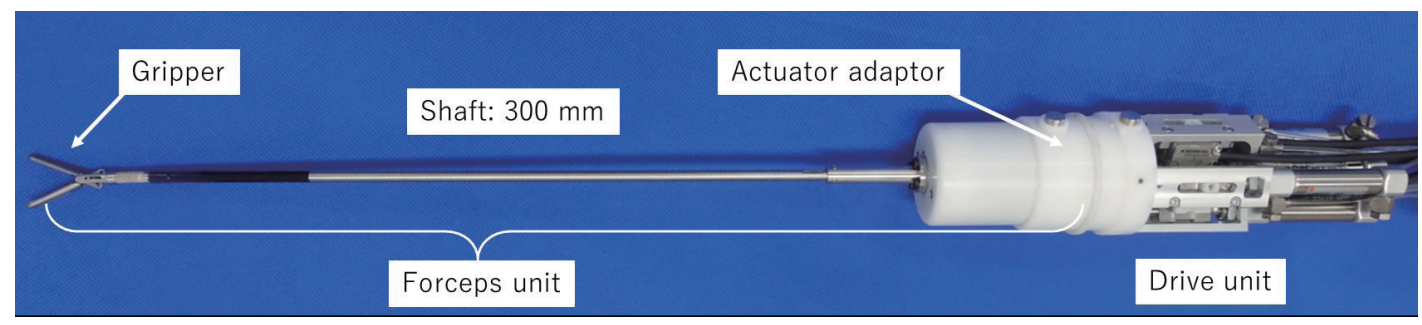

Fig. 1. (Color online) Our developed robotic forceps.

wire-tendon drive for flexible wrist control and a push-pull drive for gripper control. Pneumatic cylinders generally have an excellent power-weight ratio, so the drive unit is compact and lightweight. In addition, external force estimation can be implemented owing to the high backdrivability.

Figure 2 shows the appearance of the assembled wrist and gripper. The length of the bending segment of the flexible wrist is as compact as $10 \mathrm{~mm}$. The bending motion of the wrist is driven by four stainless steel wires $(7 \times 7$-stranded wire, diameter: $0.36 \mathrm{~mm})$. Every two opposite wires work in pairs and determine the 1-DOF bending motion driven by a tendon drive mechanism (the mechanism of driving the bending motion by the drive unit is mentioned later).

For the gripper, we used a pair of commercial grasper forceps (Autonomy Laparo-Angle instruments made by Cambridge Endo). The opening and closing motions are determined by the push-pull actuation from the central stainless steel wire $(1 \times 7$-stranded wire, diameter: $0.75 \mathrm{~mm}$ ). The slide cam at the root of the gripper segments transfers the linear motion of the driven wire into the opening and closing motions of the gripper (maximum open angle: $66^{\circ}$ ).

\subsection{Selection guideline for SEP materials}

The flexible wrist part is made of a SEP material. In this work, we selected the following seven SEP materials as candidates: polyetheretherketone (PEEK), polyphenylenesulfide (PPS), polyacetal-copolymer (POM-C), polyetherimide (PEI), polyphenylsulfone (PPSU), polysulfone (PSU), and polyethersulfone (PES). Table 1 shows the relevant properties of the SEP candidates for application in the flexible wrist joint. All candidates have been proven to have satisfactory biocompatibility for practical use as medical materials. In addition, all candidates except POM have sufficient heat resistance for autoclave sterilization (approximately $130{ }^{\circ} \mathrm{C}$ ), and they are also resistant to chemicals such as acute or alkali agents for cleaning.

In this work, we used PEEK to manufacture the wrist part because of its excellent mechanical stiffness. Other materials are also available with particular advantages suited to certain applications. PPS and PEI have good mechanical stiffness; however, low resistance to impact is an issue. PPSU, PSU, and PES are more flexible than PEEK and have good moldability, so we can reduce the manufacturing cost by simplifying the design of the flexible wrist part, which has a smaller number of slit sections and an increased wall thickness. Moreover, POM (classified as a normal engineering plastic) is also available if the forceps is intended for single use with the flexible wrist joint. 


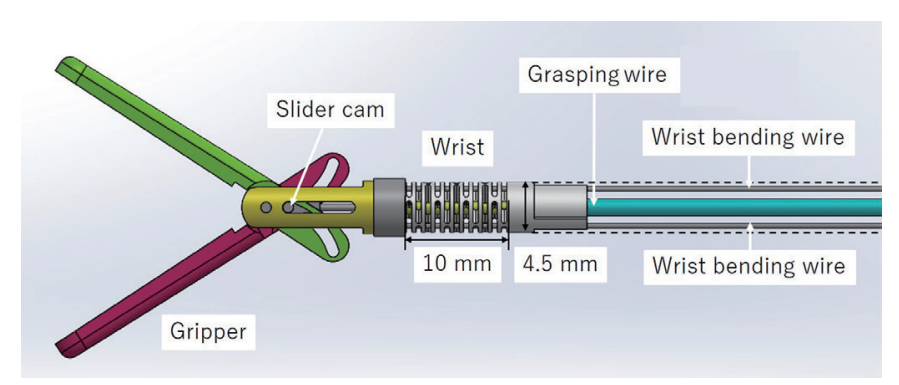

Fig. 2. (Color online) Appearance of assembled wrist, gripper, and driven wires.

Table 1

Material properties of candidate SEP materials for flexible wrist joint. ${ }^{(7,8)}$

\begin{tabular}{|c|c|c|c|c|c|c|c|}
\hline \multirow{2}{*}{ Index } & \multicolumn{7}{|c|}{ Material } \\
\hline & PEEK & PPS & POM-C & PEI & PPSU & PSU & PES \\
\hline Polymer type & \multicolumn{3}{|c|}{ Crystalline } & \multicolumn{4}{|c|}{ Amorphous } \\
\hline $\begin{array}{l}\text { Compressive } \\
\text { strength (MPa) }\end{array}$ & 105 & 96 & 65 & 92 & 66 & 75 & 108 \\
\hline $\begin{array}{l}\text { Bending strength } \\
(\mathrm{MPa})\end{array}$ & 171 & 151 & 94 & 164 & 107 & 122 & 128 \\
\hline $\begin{array}{l}\text { Bending elastic } \\
\text { modulus (MPa) }\end{array}$ & 4100 & 4000 & 2800 & 3300 & 2300 & 2600 & 2600 \\
\hline $\begin{array}{l}\text { Charpy impact } \\
\text { strength }\left(\mathrm{kJ} / \mathrm{m}^{2}\right)\end{array}$ & Not broken & 29 & Not broken & 113 & Not broken & 175 & Not broken \\
\hline $\begin{array}{l}\text { Maximum heat } \\
\text { resistance }\left({ }^{\circ} \mathrm{C}\right)\end{array}$ & 300 & 260 & 140 & 200 & 190 & 180 & 200 \\
\hline Chemical resistance & () & () & $\triangle$ & $\mathrm{O}$ & $\mathrm{O}$ & $\mathrm{O}$ & $\mathrm{O}$ \\
\hline
\end{tabular}

\subsection{Design of the PEEK flexible wrist joint}

The design and geometry of the PEEK flexible wrist are shown in Fig. 3. Twelve slits are hollowed out to realize the bending motion, the thickness of each slit is $0.4 \mathrm{~mm}$. The distance between two slits is $0.5 \mathrm{~mm}$. The connections between two slits successively shift by $45^{\circ}$ from the previous slit. Four concentric holes with a diameter of $0.5 \mathrm{~mm}$ at each slit determine the route by which the bending-motion-driven wire passes, and the central hole with a diameter of $1.8 \mathrm{~mm}$ determines the route by which the grasping-motion-driven wire (central stainless steel wire) passes.

Figure 4 shows the FEM analysis results of the stress distribution in the wrist part when a compressive force of $30 \mathrm{~N}$ is exerted at the top for outputting a grasping force. The compressive force comes from the wire tension for grasping motion. According to the gripper mechanism, if the wire is pulled by $30 \mathrm{~N}$, the grasping force will be more than $7.5 \mathrm{~N}$ in the middle of the grasper, which is certainly sufficient in laparoscopic surgery. A compressive force of more than $30 \mathrm{~N}$ is not necessary.

The analysis is conducted using Solidworks 2017. It can be seen that the compressed length is $0.48 \mathrm{~mm}$, as determined by linear static mechanics analysis. The stress at a few spots 


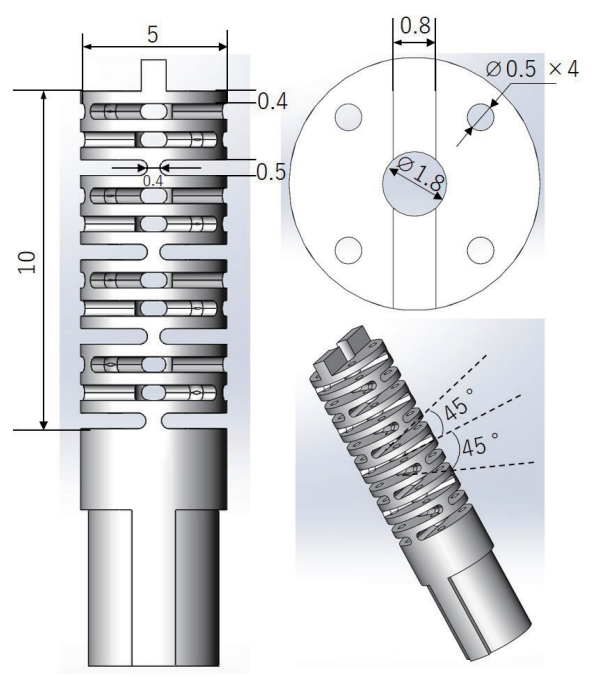

Fig. 3. Design of wrist part (made of PEEK plastic).

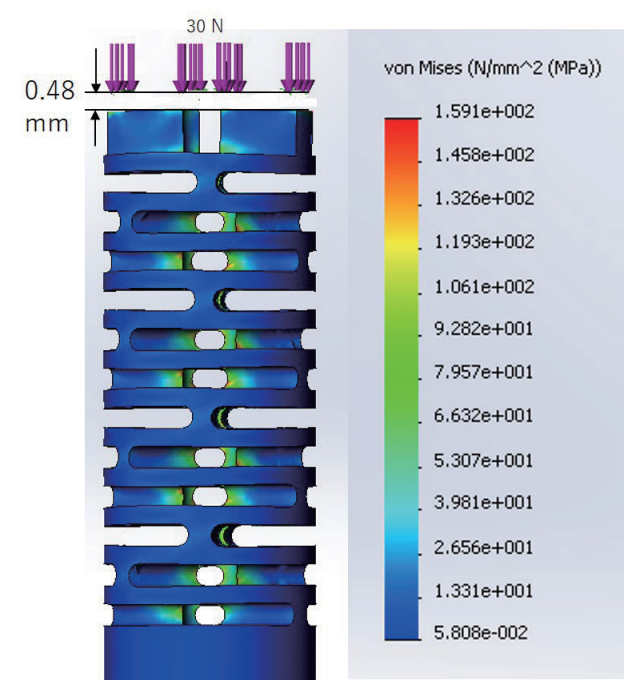

Fig. 4. (Color online) FEM analysis results of wrist part was compressed by $30 \mathrm{~N}$.

nominally exceeds the compressive strength (105 MPa according to Table 1). This result reveals that the wrist part is sufficiently durable against plastic deformation caused by an extra force due to the grasping motion.

\subsection{Pneumatic driving system}

Figure 5 shows the schematic of the pneumatic driving system in one DOF. The wires for wrist bending are connected to the corresponding bending cylinders (CJ2XB10-15Z, SMC) in the drive unit, wherein a pair of opposite cylinders retracts and extends to perform a tendon drive. Also, the grasper-driven wire is connected to a grasping cylinder (CJ2XB16-15RZ, SMC) at the center. For each cylinder, we use an analog linear encoder (ATOM4T0-100, Renishaw plc) as the position sensor to measure the position of each cylinder rod in the position control. Each cylinder is actuated by a five-port servo valve (MPYE-5-M5-010-B, FESTO) in the control unit, and two pressure sensors are attached to the two control ports of the valve, which performs the driving force control.

For the pneumatic driving force control of the cylinders, a PI controller expressed by Eq. (1) is implemented. The symbol $u$ represents the input voltage on the servo valve (considering the neutral voltage of $5.0 \mathrm{~V}), F_{\text {ref }}$ represents the desired cylinder force, $F_{\text {meas }}$ represents the measured cylinder force calculated by the pressure sensors, and $K_{p}$ and $K_{i}$ are the feedback gain parameters of the PI controller, whose values are listed in Table 2. The control period is $0.002 \mathrm{~s}$ in this system.

$$
u=K_{p} \cdot\left(F_{\text {ref }}-F_{\text {meas }}\right)+\int K_{i} \cdot\left(F_{\text {ref }}-F_{\text {meas }}\right) d t+5.0
$$

Figure 6 shows the forceps with its wrist joint bent using the pneumatic driving system. The bending angle is $67^{\circ}$, which is the mechanical bending limitation for this prototype. 


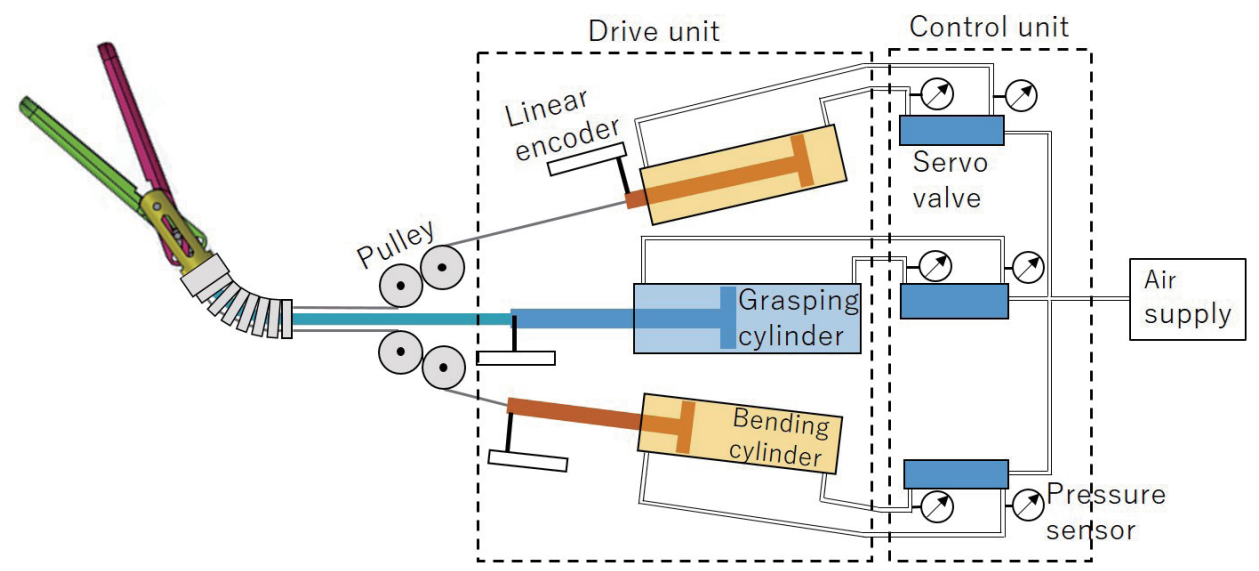

Fig. 5. (Color online) Schematic of pneumatic driving system in one DOF.

Table 2

Control parameters used in controller.

\begin{tabular}{lcc}
\hline \multirow{2}{*}{ Parameter } & \multicolumn{2}{c}{ Control object } \\
\cline { 2 - 3 } & $\begin{array}{c}\text { Wrist bending } \\
\text { cylinder }\end{array}$ & $\begin{array}{c}\text { Gripper grasping } \\
\text { cylinder }\end{array}$ \\
\hline$K_{p}(\mathrm{~V} / \mathrm{N})$ & 0.05 & 0.1 \\
$K_{i}(\mathrm{~V} / \mathrm{N} \cdot \mathrm{s})$ & 0.8 & 1.0 \\
\hline
\end{tabular}

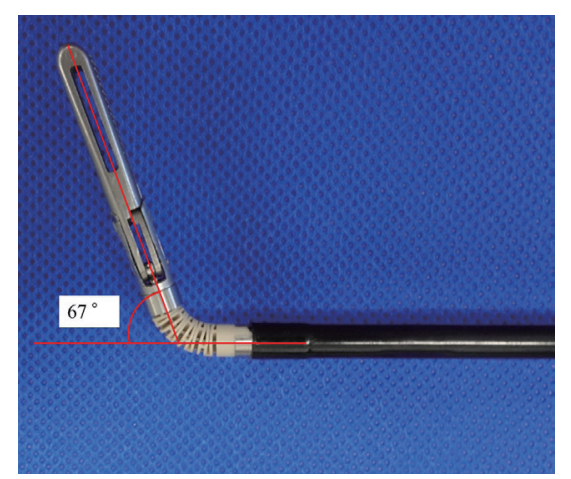

Fig. 6. (Color online) Forceps bent by $67^{\circ}$ at bending cylinder output of $20 \mathrm{~N}$.

\section{Mechanical Performance of Flexible Joint: Experiments and Results}

In this section, the experiment for evaluating the mechanical performance of the PEEK flexible wrist joint, including rigidity and durability, is introduced.

\subsection{Bending mechanics of the PEEK flexible joint}

We tested the bending mechanics of the PEEK flexible joint considering the grasper driving force. First, the forceps was set straight with an almost new PEEK wrist (bent less than 500 times). At that time, the output force of all the cylinders was controlled to be $0 \mathrm{~N}$. Then, we gradually increased the force of one of the bending cylinders to $20 \mathrm{~N}$ in steps of $1 \mathrm{~N}$. The maximum force of $20 \mathrm{~N}$ was determined to realize the maximum bending range of $67^{\circ}$. After that, we gradually reduced the cylinder force to $0 \mathrm{~N}$ in the same steps. The bending angles and corresponding cylinder forces were recorded. The bending angles were measured from the photos taken right above the flexible wrist joint as shown in Fig. 6. No grasper driving force 
was applied in this case. Figure 7 shows the change in wrist bending angle corresponding to output forces of the bending cylinder.

Next, we conducted the same experiment under the condition that the grasping cylinder retracts the grasper-driven wire to close the grasper and generate a driving force of $30 \mathrm{~N}$. In this case, a compressive load of $30 \mathrm{~N}$ due to the tension of the grasper-driven wire is exerted on the PEEK flexible joint in addition to the tension of the wrist-bending wire. Figure 8 shows the change in wrist bending angle corresponding to output forces of the bending cylinder in the presence of the grasping driving force.

On the basis of the relationship between the wrist bending angle and the bending cylinder force as the bending cylinder output increased, Figs. 7 and 8 show that when the bending cylinder output was $13 \mathrm{~N}$, the wrist bent to $50^{\circ}$ regardless of the grasper driving force. Moreover, Figs. 7 and 8 also show similar trends in the relationship between the wrist bending angle and the bending cylinder force when the bending angle was below $50^{\circ}$.

However, it became difficult to increase the bending angle in the presence of the grasping driving force and when the bending angle was beyond $50^{\circ}$. As shown in Fig. 7 (no grasping driving force), the wrist bent by $65^{\circ}$ when the bending cylinder output was $20 \mathrm{~N}$, whereas the bending angle in Fig. 8 was $61^{\circ}$ when the bending cylinder output was the same force.

When the bending cylinder output decreased, the hystereses of both cases were similar regardless of the presence or absence of the grasper driving force. (Although the starting angles of both cases were slightly different, the wrist bending angles remained almost unchanged before the bending cylinder output decreased to $10-11 \mathrm{~N}$, and when the bending cylinder output decreased to $6 \mathrm{~N}$, the wrist bending angles of both cases were restored to $50^{\circ}$ ).

The main reason for the hysteresis is considered to be the static friction of the actuation transmission mechanism, i.e., the friction produced by the cylinder piston and the wire and its guiding route. Taking the cylinder force increasing process in Fig. 7 as an example, the bending angle was $50^{\circ}$ when the bending cylinder force was $13 \mathrm{~N}$, while in the cylinder force decreasing process, the bending angle was restored back to $50^{\circ}$ when the bending cylinder force was $6 \mathrm{~N}$. An extra force of about $7 \mathrm{~N}$ interfered with the bending wire from bending restored to $50^{\circ}$.

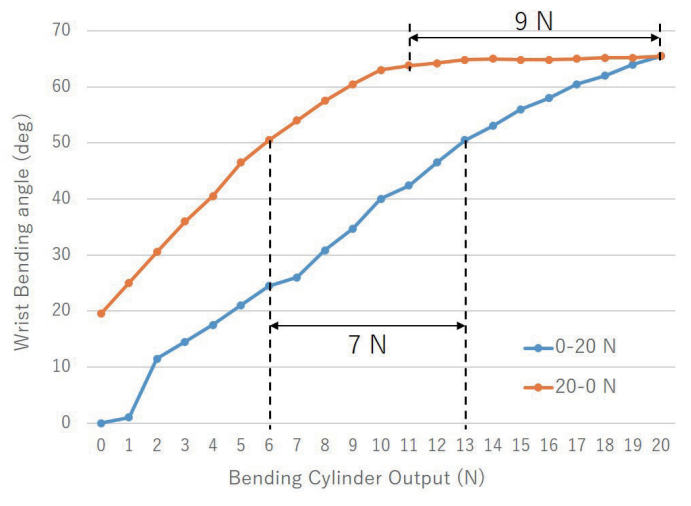

Fig. 7. (Color online) Relationship between bending cylinder force and wrist bending angle without grasper driving force.

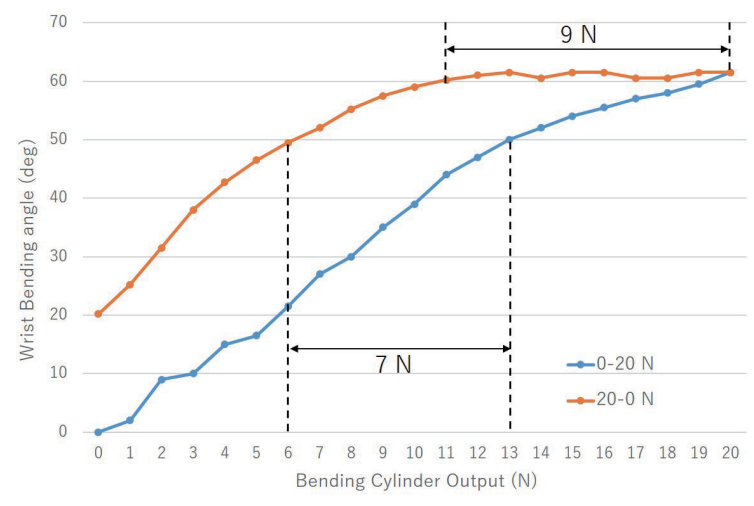

Fig. 8. (Color online) Relationship between bending cylinder force and wrist bending angle with grasper driving force of $30 \mathrm{~N}$. 
Additionally, Figs. 7 and 8 show that the cylinder output differences between the increasing and decreasing processes for making the wrist part bend at the same angle were 6 or $7 \mathrm{~N}$ when the bending angle was less than $50^{\circ}$ and $9 \mathrm{~N}$ at the maximum bending angle. Hence, the magnitude of the static friction was estimated to be 6-9 N.

Regarding the variance of the bending angle according to the cylinder force after bending more than $50^{\circ}$ in the cases with and without the grasper driving force, we considered that this variance was caused by the compression of the PEEK wrist joint. In the case without a grasper driving force, the slits of the PEEK wrist joint on the bending side began to come in contact with each other at a bending angle of about $60^{\circ}$. On the other hand, when the wrist part was slightly compressed with the grasper driving force, the slits on the bending side began to come in contact and squeeze each other at a bending angle of about $50^{\circ}$. Then, the bending cylinder had to output extra squeezing force, which led to a smaller bending angle of the wrist than in the case without the grasper driving force. According to these observations, the SEP flexible joint combined with the grasper driving controller should be designed and implemented so as not to cause this type of mechanical interference.

\subsection{Compression rigidity test}

As discussed in the previous section, the grasping cylinder pulls the central wire to generate a grasping force, causing an additional compressive force to be exerted on the wrist part. Since it will lead to an error in the position control, the compression of the wrist part should also be evaluated.

In the experiment, we kept the force of the four bending cylinders at $0 \mathrm{~N}$ and gradually increased the grasping cylinder force to $30 \mathrm{~N}$ in steps of $2 \mathrm{~N}$. The length of the wrist part and the corresponding cylinder force were measured and recorded.

Figure 9 shows the relationship between the grasping cylinder force and the wrist length. The experimental result reveals that when the compression force increases to $30 \mathrm{~N}$, the wrist part is compressed by $0.62 \mathrm{~mm}$. On the other hand, the result of the FEM analysis shows the

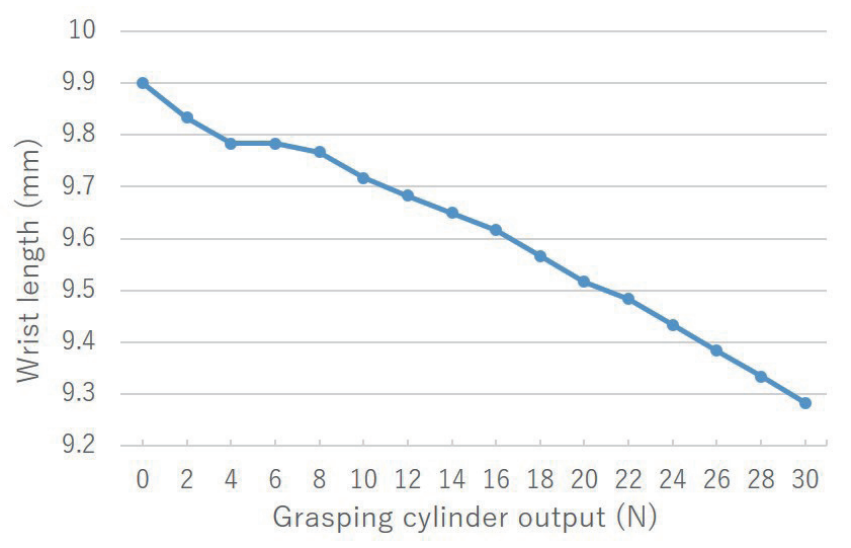

Fig. 9. (Color online) Relationship between grasping cylinder output and wrist length. 
wrist part compression of $0.48 \mathrm{~mm}$ under the same compressive load (Sect. 2.2 and Fig. 4), which can approximately predict the compressive deformation of the PEEK wrist part.

The compression of the wrist joint leads to a position error of the forceps tip. However, the compression length is measurable in real time using the positions of the four bending cylinders. Thus, the position error might be compensated by the control system of the whole surgical robot arm.

\subsection{Bending durability test}

Robotic forceps should have a long life. Here, we conducted experiments to examine the following items regarding durability performance: the durability of the developed forceps with the PEEK flexible wrist to bending fatigue and whether the bending characteristics remain unchanged. We investigated the relationship described in Sect. 3.1.

In the experiment, the two bending cylinders are made to output driving forces following a sine function reference (amplitude: $20 \mathrm{~N}$, frequency: $1 \mathrm{~Hz}$ ) to repeatedly perform a one-DOF bending motion of the flexible wrist. Thus, the wrist joint continuously bends on both sides at a bending angle amplitude of about $65^{\circ}$. During the experiment, we paused the bending upon completing 1000, 2000, 3000, 5000, and 10000 repetitions, and performed the experiment conducted in Sect. 3.1 without the grasper driving force.

The relationships between the bending cylinder force and the wrist bending angle after each bending repetition time set are plotted in Fig. 10. The results show that the bending characteristics of the PEEK flexible wrist do not markedly change. With regards to the variation between the bending angle-cylinder output curves, we considered that it was caused by the static friction of the actuation transmission mechanism resulting in the hysteresis in Figs. 7 and 8 . As to the curves after 2 and $10 \mathrm{~K}$ bending by which the largest variation appears, Fig. 10 shows that the cylinder output difference between the two curves for making the wrist part bend to the same angle was $4-5 \mathrm{~N}$, which is within the static friction range $(6-9 \mathrm{~N})$ discussed in Sect. 3.1. Additionally, cracks and large plastic deformations did not occur on the wrist part.

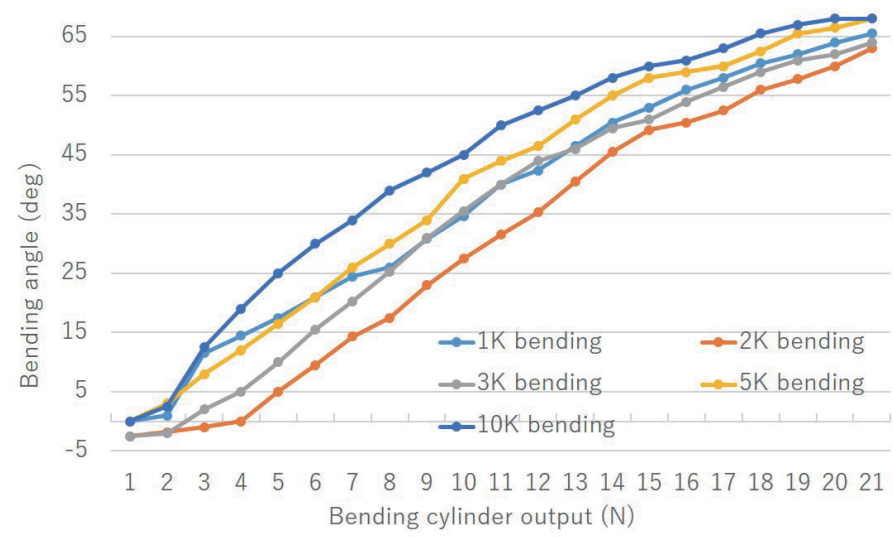

Fig. 10. (Color online) Relationship between bending cylinder output and bending angle after 1-10 thousands of bending repetitions. 


\subsection{Bending rigidity test}

A surgery assistant robot is often required to exert an operation force to the target object such as those produced by touching and lifting. In these cases, the bending motion of the wrist joint should be rigid enough to transmit the necessary force. A large additional deformation due to the counterforce from the environment is unacceptable.

We conducted an experiment to confirm this force-exertion performance. Figure 11 shows the apparatus used in the experiment. The motion of the tip of the forceps in one direction was restrained by a jig fixed on a force sensor (CFS018CA101U, Leptorino Corp.). At the beginning, the output forces of the four bending cylinders were $0 \mathrm{~N}$. Then, for the bending cylinder that drives the wrist joint to bend in the restrained direction, we increased the output force to $26 \mathrm{~N}$ in steps of $2 \mathrm{~N}$. This experiment was conducted in the $Z$-axis and $X$-axis directions, as shown in Fig. 11.

Figures 12 and 13 show the forces exerted on the environment measured by the force sensor in the two directions and the state of the wrist joint. The linearity between the cylinder force and the forceps output force reveals that the PEEK wrist joint is rigid enough to transmit the actuation force to the environment. The maximum value of about $1.2 \mathrm{~N}$ was measured in both directions. If we apply greater driving forces, the forceps can output greater forces, but at the same time, the compressive deformation of the wrist joint may also become larger. In this experiment, when the actuator force increased, a local deformation occurred at the connecting point between the wrist joint and the grasper. This deformation was due to the attachment backlash between the wrist and the grasper derived from the mechanical design.

\section{Discussion}

The experiments in Sect. 3 demonstrated that the performance characteristics in terms of the durability and rigidity of the PEEK flexible wrist joint are basically feasible for robotic surgery. In comparison with the performance observed using the flexible forceps introduced by

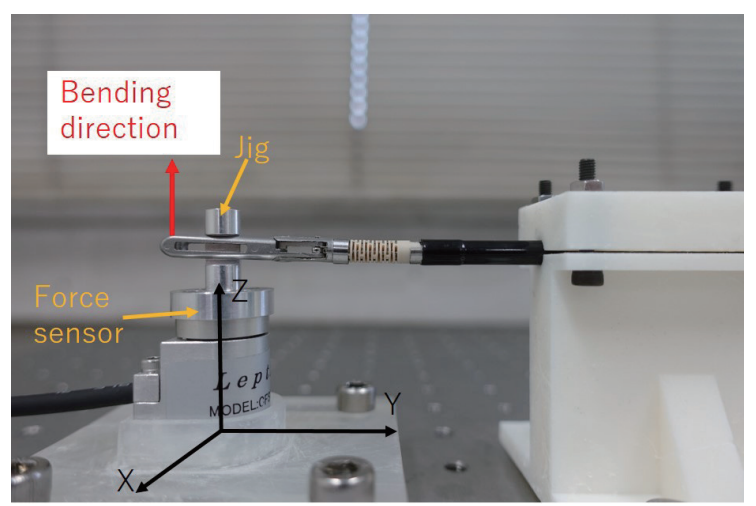

(a)

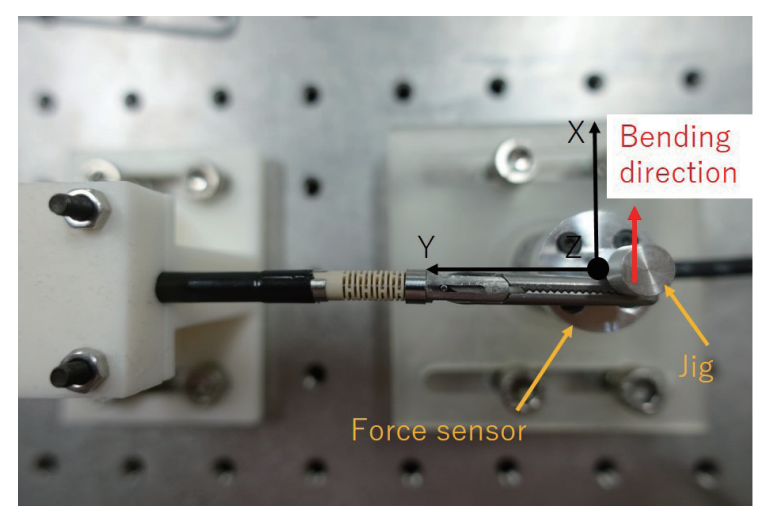

(b)

Fig. 11. (Color online) Apparatus used in bending rigidity experiment: (a) $Z$-axis and (b) $X$-axis directions. 


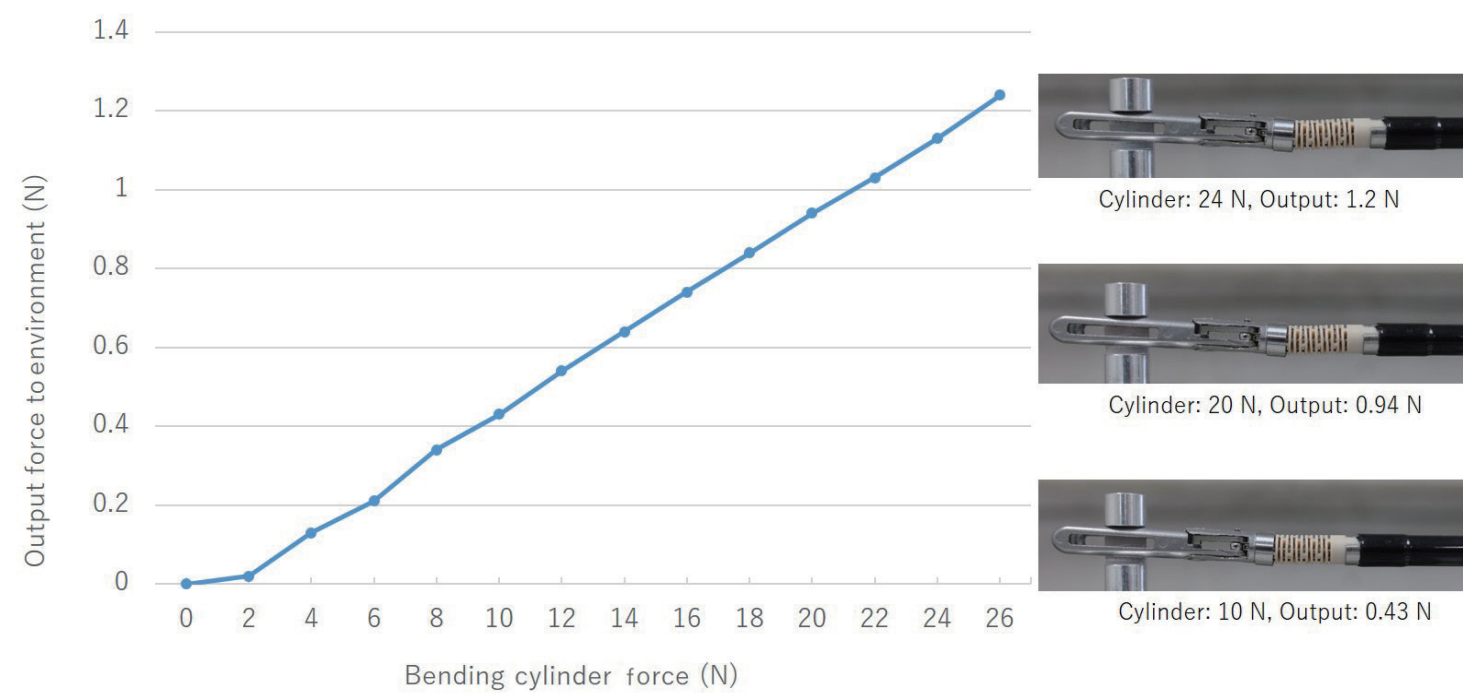

Fig. 12. (Color online) Force exerted on environment as bending cylinder output increased ( $Z$-axis direction).

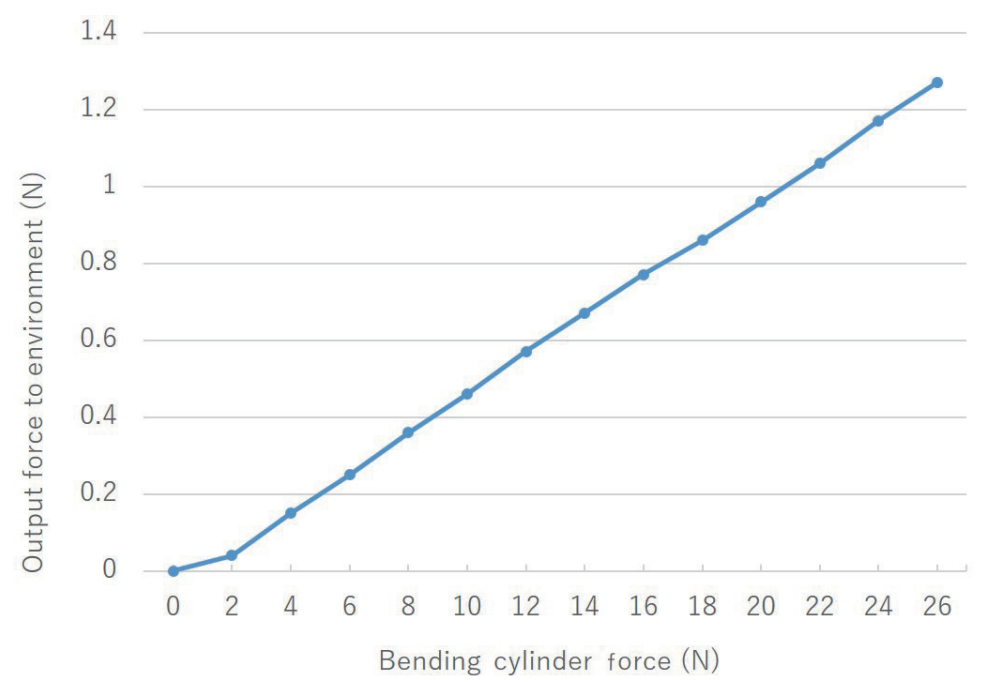

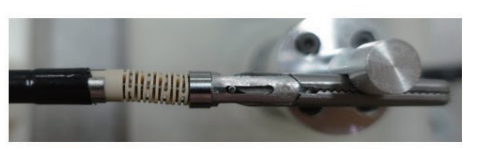

Cylinder: $26 \mathrm{~N}$, Output: $1.27 \mathrm{~N}$

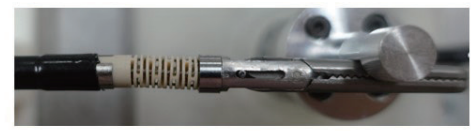

Cylinder: $20 \mathrm{~N}$, Output: $0.96 \mathrm{~N}$

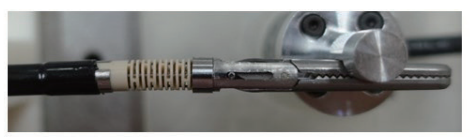

Cylinder: $10 \mathrm{~N}$, Output: $0.46 \mathrm{~N}$

Fig. 13. (Color online) Force exerted on environment as bending cylinder output increased ( $X$-axis direction).

Miyazaki et al., ${ }^{(4)}$ which has similar outer diameter and bending range of the wrist joint to our work, the length of the wrist part in this study is only $12 \mathrm{~mm}$, while the wrist part of the device introduced in Ref. 4 is $28 \mathrm{~mm}$. This means that our proposed forceps has much dexterity to work well in a narrow space. With regards to the performance in terms of output force, our proposed forceps is powerful with respect to the bending force $(1.2$ to $1.0 \mathrm{~N})$ and grasping force $(7.5$ to $0.6 \mathrm{~N})$.

However, several improvements are still necessary to enhance the performance. First, the friction between the driven wire and the other parts should be reduced. This friction interferes with the bending angles when the cylinder outputs the same forces. The effect of the friction 
should be dynamically compensated in the force control. With regards to position control, we can use the position sensors to measure the real bending angle and apply this information into the control loop. Second, the compression rigidity of the wrist part should also be enhanced to keep the maximum bendable angle and reduce the forceps position error in position control. Third, the maximum bending force without a large deformation should be enhanced. The experimental results in Sect. 3.4 show that the maximum bending force without a large deformation is $1.2 \mathrm{~N}$. A force of this magnitude is sufficient for surgery operations such as cutting, resection, peeling, and suturing, but insufficient to lift or hold large organs.

\section{Conclusion}

We presented a pair of robotic forceps with a flexible wrist joint made of PEEK plastic, a type of SEP material. The bending and grasping motions of this forceps were realized by wire actuation driven by pneumatic cylinders located away from the tip. The results of the performance evaluation experiments clarified the mechanical properties of the wrist joint. Knowledge of the relationship between the bending actuation output and the bending angle was helpful for building a simple dynamic model for the position controller. However, this relationship changed after bending more than $50^{\circ}$ owing to the joint compression caused by the grasper driving force of $30 \mathrm{~N}$, with which the PEEK wrist joint was compressed by $0.62 \mathrm{~mm}$. Regarding durability, the bending characteristics did not markedly change even after repeating the wrist joint bending 10000 times without the grasper driving force. With respect to bending rigidity, the PEEK wrist joint can withstand an external force of more than $1.2 \mathrm{~N}$ in the middle of the grasper and maintain its shape when in contact with the environment. In conclusion, the robotic forceps with a PEEK wrist joint exhibits satisfactory performance and have the potential for use in robotic surgery.

In our future work, we will improve the design of the wrist part on the basis of experimental results, then build a position control system with external force estimation using a pneumatic drive, and evaluate its performance in terms of practical surgical applications.

\section{Acknowledgments}

This work was supported by the collaborative research fund of RIVERFIELD Inc.

\section{References}

1 J. Ding, K. Xu, and R. Goldman: Proc. 2010 IEEE Int. Conf. Robotics and Automation (ICRA, 2010) 1053.

2 D. Haraguchi, K. Tadano, and K. Kawashima: Proc. 2011 IEEE/RSJ Int. Conf. Intelligent Robots and Systems (IROS, 2011) 931.

3 Y. Hu, W. Li, L. Zhang, and G. Yang: IEEE Robotics and Automation Letters. 4 (2019) 2. https://doi. org/10.1109/LRA.2019.2896883

4 R. Miyazaki, T. Kanno, and K. Kawashima: Int. J. Med. Rob. Comput. Assisted Surg. 15 (2019) 3. https://doi. org/10.1002/rcs.1983

5 W. Cheng, K. Song, Y. Di, E. Zhang, Z. Qian, S. Kanagaratnam, M. Moser, W. Luo, and W. Zhang: J. Med. Biol. Eng. 33 (2013) 337. 
6 A. Schmitz, S. Treratanakulchai, P. Rayne, and G. Yang: Proc. 2019 IEEE Int. Conf. Robotics and Automation (ICRA, 2019) 379. https://doi.org/10.1109/ICRA.2019.8793480

7 E. Baur, T. A. Osswald, and N. Rudolph: Plastic Handbook: The Resource for Plastics Engineers. (Carl Hanser Verlag, Munich, 2019) 2nd ed., Chap. 3.

8 Ensinger: https://www.ensingerplastics.com/en/medical

\section{About the Authors}

Dongbo Zhou received his B.S. degree from Nanchang Hangkong University, China, in 2009 and his M.S. degree from The University of Tsukuba, Japan, and Ph.D. degree from Tokyo Institute of Technology, Japan, in 2011 and 2019, respectively. From 2019, he became a specialappointed assistant professor at Tokyo Institute of Technology, Japan. His research interests are in robotics, haptics, and mechanics. (zhou.d.aa@m.titech.ac.jp)

Kiyoshi Sanada graduated from the Mechanical Engineering Course of the Special Engineering College of Kogakuin University, Japan, in 1995. He is currently working for RIVERFIELD Inc., Japan. (k-sanada.rfc@riverfieldinc.com)

Daisuke Haraguchi received his B.S. degree from National Defense Academy, Japan, in 2003 and his M.S. and Ph.D. degrees from Tokyo Institute of Technology, Japan, in 2010 and 2013, respectively. From 2014 to 2018, he was the CEO of RIVERFIELD Inc., Japan, developing and commercializing surgical robot products. In 2019, he became a research associate professor at Tokyo Institute of Technology. (haraguchi.d.aa@m.titech.ac.jp) 